\title{
Many-Body Effects and Bandgap Renormalization in H-Shaped Quantum Wires
}

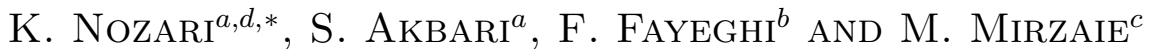 \\ ${ }^{a}$ Department of Physics, Faculty of Basic Sciences, University of Mazandaran \\ P.O. Box 47416-1467, Babolsar, Iran \\ ${ }^{b}$ Department of Physics, Payam-e Nour University \\ P.O. Box 919, Mashad, Iran \\ ${ }^{c}$ Department of Physics, Iran University of Science and Technology, Tehran, Iran \\ ${ }^{d}$ Nano and Biotechnology Research Group \\ Faculty of Basic Sciences, University of Mazandaran, Iran
}

(Received September 16, 2008; in final form December 17, 2008)

\begin{abstract}
In this paper, bound states energies and corresponding wave functions of H-shaped quantum wires are calculated numerically in the presence of the external magnetic and electric fields. This analysis was done within the Landau gauge. With a suitable definition of the external confinement potential, we present a numerical algorithm to calculate the profile of the probability distribution of charge carriers. Our analysis shows that in the presence of the external electric and magnetic fields, bound state properties of the carriers are sensitive functions of an asymmetric parameter $a=W_{x} / W_{y}$ which measures the relative width of the quantum well in two directions. We also study many-body effect of the bandgap renormalization in this quasi-one-dimensional system within the dynamical random phase approximation in its leading order.
\end{abstract}

PACS numbers: 71.35.Ee, 71.45.Gm

\section{Introduction}

A highly dense electron-hole plasma can be generated in a wide variety of semiconductors by the optical pumping. The band structure and the optical properties of highly excited semiconductors generally differ from those calculated for non-interacting electron-hole pairs due to many-body exchange-correlation effects arising from the electron-hole plasma $[1,2]$. In recent years, quasi-one-dimensional semiconductor quantum wires $(\mathrm{QW})$ have been fabricated in a variety of the geometric shapes with atomic scale definition, and their optical properties have been studied for potential device applications such as semiconductor lasers [3, 4]. Recently, different geometries of quantum wires, such as rectangular, V-shaped, L-shaped and T-shaped quantum wires have been fabricated and some of their electronic properties have been studied [4]. In addition, various experimental techniques for fabrication and growth of these structures have been developed [3-5]. Square quantum well wires have been studied by $\mathrm{Hu}$ and Das Sarma [6].

* corresponding author; e-mail: knozari@umz.ac.ir
They have calculated the value of the bandgap renormalization (renormalization of the fundamental gap structure due to many-body exchange-correlation effects) for this structure within GW approximation. Excitonic effects in semiconductor quantum wires have been studied by Goldoni et al. [7]. They have studied the effects of the Coulomb interaction on the linear and nonlinear optical properties of both V-shaped and T-shaped semiconductor quantum wires. Wang and Das Sarma have proposed an elegant framework for numerical studies of the carrier induced many-body effects on the excitonic optical properties of highly photoexcited one-dimensional quantum wire systems $[8,9]$. Hwang and Das Sarma have investigated the dynamical self-energy corrections of electron-hole plasma due to electronelectron and electron-phonon interactions at the band edges of a quasi-one-dimensional photoexcited electronhole plasma within the GW approximation [10, 11]. Rinaldi and Cingolani have studied the optical properties of quasi-one-dimensional quantum structures, specially the case of V-shaped quantum wires [12]. Bener and Haug have considered plasma-density dependence of the optical spectra for quasi-one-dimensional quantum well wires [13]. Tanatar has studied the band gap renor- 
malization in quasi-one-dimensional systems in a simple plasmon-pole (quasi-static) approximation [14, 15]. Güven et al. have studied the bandgap renormalization in quantum wire system by incorporation of the dynamical correlations and multisubband effects [16]. Luttinger liquid behavior of a semiconductor quantum wire has been studied by Bellucci and Onorato [17]. They have also studied the effects of the magnetic field on low dimensional electron systems focusing on the Luttinger liquid behavior in a quantum wire [18]. In addition, they have studied also the ballistic electron transport in a quantum wire under the action of an electric field [19] and quenching of the spin Hall effect in ballistic nanojunctions [20]. As an application, they have studied the transport through a double barrier in a large radius carbon nanotube with transverse magnetic field [21]. Many particle aspects of a semiconductor quantum wire within an improved random phase approximation has been studied by Ashraf and Sharma [22]. They have considered structure factor, pair distribution function, screened impurity potential, and density of the screening charge and exchange and screened exchange energies within an improved random phase approximation.

On the other hand, T-shaped and L-shaped quantum wires have been studied by some authors. For example, Sedlmaier and his co-workers have studied the bandgap renormalization of the modulation doped T-shaped quantum wires. They have presented a self-consistent electronic structure calculation for this device [23]. Using a density functional theory, Stopa has calculated the electronic structure of a modulation doped and gated T-shaped quantum wire [24]. Szymanska et al. have studied the excitons in T-shaped quantum wires [25]. They have calculated energies and oscillator strength for radiative recombination and two particle wave functions for ground state exciton in a T-shaped quantum wire. Lin et al. have found the dependence of the bound states of L-shaped and T-shaped quantum wires to some asymmetric parameter in an inhomogeneous magnetic field [26]. They have proposed a simple model to explain the behavior of the magnetic dependence of the bound state energies both in the weak and strong field regions. Nozari and Madadi have studied numerically the many-body properties and the bandgap renormalization of V-shaped and T-shaped quantum wires within the dynamical random phase approximation [27, 28]. Ballistic transport through coupled T-shaped quantum wires has been studied by Lin et al. [29].

As another possible geometry of low dimensional systems, H-shaped electronic systems have been considered and their many-body electronic properties have been studied by some authors. These type of quasi-one-dimensional system could be imagined as two T-shaped quantum wire joint together. Shin and co-workers have studied quantum transport in the $\mathrm{H}$-shaped quantum wire and also a ring structure [30]. They have studied numerically the transport properties of H-shaped quantum wire structure by using the mode matching tech- nique. They have reported the existence of anomalous Hall resistance plateaus in this structure with relatively low magnetic fields as precursors of integer Hall plateaus. Hankiewicz et al. have studied the manifestation of the spin Hall effect in a two-dimensional electronic system with the Rashba spin-orbit coupling via dc-transport measurements in a realistic H-shaped structure [31]. Designing of H-shaped micromechanical structure has been studied by Arhaug and Soeraasen [32].

Our investigation shows that there is no other concrete study of the $\mathrm{H}$-shaped quantum wire structure in the existing literature. Obviously, an analytical-numerical study of this special structure is important to fill the existing gap. Especially, our investigation in the literature shows that bound states of H-shaped quantum wires and many-body effects such as bandgap renormalization of these low dimensional systems have not been studied yet. Therefore, in this paper we consider the geometry of H-shaped quantum wires and by a suitable analytical definition of quasi-one-dimensional H-shaped confinement potential, we propose a numerical scheme for calculating the bound states energies and wave functions of charge carriers in the presence of external electric and magnetic fields and within the Landau gauge. Application of the external electric and magnetic field is itself an important step in this regard since in electronic devices based on these structure we cannot neglect the effects of these fields. We obtain the profile of charge carriers distribution (probability distribution) in the presence of external electric and magnetic fields. As an important nonlinear optical effect, the many-body exchange-correlation induced bandgap narrowing in this type of the quantum wire will be studied within the leading order dynamical random phase approximation. Then we compare the results of our calculations in quasi-static and GW approximation.

This paper is organized as follows: Sect. 2 is devoted to formulation of the problem and definition of the confinement potential mathematically. In Sect. 3 we give a short but complete overview of the theory of bandgap renormalization in a general quasi-one-dimensional semiconductor system. Some numerical details are given at the end of this section. Section 4 provides numerical results of our study and their interpretation, while the numerical scheme of our calculations based on the finite difference algorithm is presented in Appendix. The results of each step are shown by figures. Finally, summary and conclusions are given in Sect. 5 .

\section{The setup}

The geometry of a typical H-shaped quantum wire is shown in the Fig. 1. This structure can be thought as two distinct T-shaped quantum wire joint together. The typical values of $W_{x}$ and $W_{y}$ are of the order of 50-100 nm. We study many-body effects originating from the optical non-linearities in this quasi-one-dimensional system in the presence of the external electric and magnetic fields. 


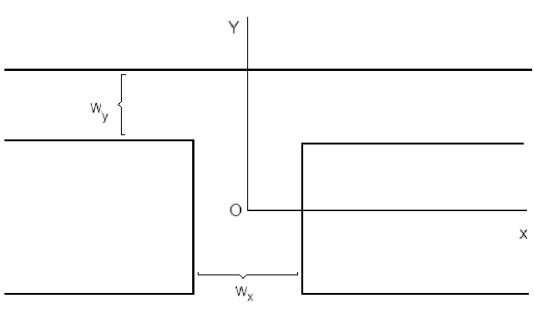

Fig. 1. The geometry of the H-shaped quantum wire.

The electric field is assumed to be directed along the $x$ axis and its typical value is of the order of a few volt per centimeter, $\mathrm{V} / \mathrm{cm}$. The presence of the magnetic field is in such a way that the Landau gauge, $\boldsymbol{A}=(0, B x, 0)$ is satisfied. The Hamiltonian of an electron in this configuration can be written as follows:

$$
H=\frac{1}{2 m}\left[p_{x}^{2}+\left(p_{y}-e B x\right)^{2}\right]-e E x,
$$

where $e$ and $m$ are electron's charge and mass, respectively. The Schrödinger equation of this electron with wave function $\Psi(x, y)$ can be written as follows:

$$
\begin{aligned}
& -\left(\frac{\partial^{2}}{\partial x^{2}}+\frac{\partial^{2}}{\partial y^{2}}-\frac{2 \mathrm{i} e B}{\hbar} x \frac{\partial}{\partial y}\right) \Psi(x, y) \\
& +\left(\frac{e B}{\hbar}\right)^{2} x^{2} \Psi(x, y)-\frac{2 m e E}{\hbar^{2}} x \Psi(x, y)= \\
& \quad \frac{2 m}{\hbar^{2}} \lambda \Psi(x, y),
\end{aligned}
$$

where $\lambda$ stands for eigenvalues of electron energy, $E$ represents the value of the electric field and $B$ is the value of the magnetic field. To solve this eigenvalue problem, we need the boundary conditions which are given by the external confinement potential. The geometry of H-shaped quantum wire as shown in Fig. 1, suggests the following definition of confinement potential which contains appropriate boundary conditions based on H-shaped geometry

$$
\begin{aligned}
& V(x, y)= \\
& \left\{\begin{array}{l}
0 \text { if }\left\{\begin{array}{l}
-\infty<x<+\infty, \quad-\frac{3}{2} W_{y} \leq y \leq-W_{y}, \\
-\infty<x<+\infty, W_{y} \leq y \leq \frac{3}{2} W_{y}, \\
-\frac{W_{x}}{2} \leq x \leq \frac{W_{x}}{2}, \quad-W_{y} \leq y \leq W_{y},
\end{array}\right. \\
\infty \text { elsewhere. }
\end{array}\right.
\end{aligned}
$$

It should be emphasized that the H-shaped geometry is the transverse section of the wire and that the free carrier propagation direction is perpendicular to the plane of Fig. 1. With this definition of the confinement potential which provides the required boundary conditions, we solve Eq. (2) numerically to find eigenvalues and eigenfunctions of bound states of electron in the presence of the electric and magnetic fields. Our numerical strategy is based on the finite difference algorithm and is presented in Appendix. The results of these calculations for different external field configurations and variety of the possibilities are shown in Figs. 2, 4, 6, 7 and 9 and will be interpreted in Sect. 4. Also energy profiles of these single-particle states are shown in Figs. 3, 5, 8 and 10 for different electromagnetic (EM) field configurations and different asymmetry parameter. The wave functions obtained in this section will be used to study the bandgap renormalization of the $\mathrm{H}$-shaped quantum wire in the next section.

\section{Many-body and nonlinear optical effects in H-shaped quantum wires}

There are several nonlinear optical and many-body effects in the quasi-one-dimensional semiconductor systems which originate from the exchange-correlation effects in a dense excited plasma. One of the most important many-body effect in a high density electron-hole plasma is a density-dependent renormalization of the fundamental band gap of the semiconductor which affects an increasing absorption in the spectral region below the lowest exciton resonance. The exchange-correlation correction of the fundamental band gap due to the presence of free carriers (electrons in the conduction band and holes in the valence band) in the system is referred to as the band gap renormalization effect. Optical nonlinearities, which are strongly influenced by the screened Coulomb interaction in the electron-hole plasma, are typically associated with the band gap renormalization phenomenon. In which follows, we use the two-band (a conduction and a valence band) model to study the one-dimensional electron-hole system. We neglect the effects of higher subbands and degeneracies in valence bands. We assume that electrons and holes densities are constant in time. We also assume that only one kind of electron and one kind of hole with parabolic isotropic dispersion exist. This should be an adequate approximation for calculating the renormalization at the band edge especially for semiconductor structure such as GaAs. In this situation, Hamiltonian of the system can be written as (see for instance $[8,9,33]$ ):

$$
\begin{aligned}
H & =\sum_{k}\left[\left(E_{\mathrm{g}}^{0}+\frac{k^{2}}{2 m_{\mathrm{e}}} c_{k}^{\dagger} c_{k}+\frac{k^{2}}{2 m_{\mathrm{h}}} d_{k}^{\dagger} d_{k}\right)\right] \\
& +\frac{1}{2 L} \sum_{k, k^{\prime}, q}\left[V_{\mathrm{c}, \mathrm{ee}}(q) c_{k-q}^{\dagger} c_{k^{\prime}+q}^{\dagger} c_{k^{\prime}} c_{k}\right. \\
& +V_{\mathrm{c}, \mathrm{hh}}(q) d_{k-q}^{\dagger} d_{k^{\prime}+q}^{\dagger} d_{k^{\prime}} d_{k} \\
& \left.+V_{\mathrm{c}, \mathrm{eh}}(q) c_{k-q}^{\dagger} c_{k} d_{k^{\prime}+q}^{\dagger} d_{k^{\prime}}\right] .
\end{aligned}
$$

In this equation which contains all information about the one-dimensional system under consideration, $c_{k}$ and $c_{k}^{\dagger}$ are annihilation and creation operators for conduction electrons, respectively. Also $d_{k}$ and $d_{k}^{\dagger}$ are annihilation and creation operators for valence band holes. $E_{\mathrm{g}}^{0}$ is the direct band gap between the top of the valence band and the bottom of the conduction band. $V_{\mathrm{c}, i j}$ show the pos- 
sible three Coulomb interactions between electrons and holes. Let us note that the two first interactions lead to electron-hole quasi-particle self-energies while the third one leads to the production of excitonic bound states. Let us note also that this Hamiltonian consists of spin effects, although spin index is not included explicitly.

The Coulomb interaction matrix element in one-dimensional quantum wire is given by the following relation [8]:

$$
\begin{aligned}
& V_{\mathrm{c}, i j}(q)=\frac{e^{2}}{\varepsilon_{0}} \int_{-\infty}^{+\infty} \mathrm{d} x \mathrm{~d} x^{\prime} \int_{-\infty}^{+\infty} \mathrm{d} y \mathrm{~d} y^{\prime} \int_{-\infty}^{+\infty} \mathrm{d} z \\
& \quad \times \frac{\mathrm{e}^{-\mathrm{i} q z}\left|\phi_{i}(x, y)\right|^{2}\left|\phi_{j}\left(x^{\prime}, y^{\prime}\right)\right|^{2}}{\sqrt{z^{2}+\left(y-y^{\prime}\right)^{2}+\left(x-x^{\prime}\right)^{2}}} \\
& =\frac{2 e^{2}}{\varepsilon_{0}} \int_{-\infty}^{+\infty} \mathrm{d} x \mathrm{~d} x^{\prime} \int_{-\infty}^{+\infty} \mathrm{d} y \mathrm{~d} y^{\prime}\left|\phi_{i}(x, y)\right|^{2} \\
& \quad \times\left|\phi_{j}\left(x^{\prime}, y^{\prime}\right)\right|^{2} K_{0}\left(q \sqrt{\left(x-x^{\prime}\right)^{2}+\left(y-y^{\prime}\right)^{2}}\right)
\end{aligned}
$$

where $\phi_{i}(x, y)$ is the quantum wire confinement wave function for the lowest eigenstate of electrons or holes. The exact form of these eigenfunctions depends on the geometry and details of confinement potential. In our case with $\mathrm{H}$-shaped confinement potential, these eigenfunctions will be calculated numerically based on the algorithm presented in the previous section. $K_{0}(x)$ is the zeroth-order modified Bessel function of the second kind. In the setup of our one-dimensional quantum system, we have assumed that carriers are free to move in the $z$ direction but $x$ and $y$ are directions of confinement.

Band gap renormalization in the quasi-static approximation is given by $[14,15]$ :

$$
\begin{aligned}
& \Delta_{i}(k)=\sum_{k^{\prime}}\left\{-V_{\mathrm{s}}\left(k-k^{\prime}\right) n_{i}\left(\varepsilon_{i, k}\right)\right. \\
& \left.+\frac{1}{2}\left[V_{\mathrm{s}}\left(k^{\prime}\right)-V_{\mathrm{c}}\left(k^{\prime}\right)\right]\right\},
\end{aligned}
$$

where

$$
V_{\mathrm{s}}(k) \equiv V_{\mathrm{s}}(k, \omega=0)=\frac{V_{\mathrm{c}}(k)}{\varepsilon(k, \omega=0)}
$$

is the statically screened Coulomb interaction and $n_{i}\left(\varepsilon_{i, k}\right)$ is the fermion momentum distribution function

$$
n_{i}\left(\varepsilon_{i, k}\right)=\frac{1}{\mathrm{e}^{\beta\left(\varepsilon_{i, k}-\mu_{i}\right)}+1},
$$

where $\varepsilon_{\mathrm{e}, k} \equiv \frac{k^{2}}{2 m_{\mathrm{e}}}+E_{\mathrm{g}}^{0}$ and $\varepsilon_{\mathrm{h}, k} \equiv \frac{k^{2}}{2 m_{h}}$ are the bare energies of electron and hole in their respective bands and $\mu_{i}$ is the chemical potential. $\varepsilon(k, \omega)$ as the dynamical dielectric function is defined as follows:

$$
\begin{aligned}
& \varepsilon(k, \omega)=1-V_{\mathrm{c}}(k) \Pi_{\mathrm{e}}^{0}(k, \omega)-V_{\mathrm{c}}(k) \Pi_{\mathrm{h}}^{0}(k, \omega)= \\
& 1-V_{\mathrm{c}}(k) \sum_{i=\mathrm{e}, \mathrm{h}} \frac{m_{i}}{\pi k} \\
& \quad \times \ln \left(\frac{\omega^{2}-\left(\left(k^{2} / 2 m_{i}\right)-k v_{\mathrm{F}, i}\right)^{2}}{\omega^{2}-\left(\left(k^{2} / 2 m_{i}\right)+k v_{\mathrm{F}, i}\right)^{2}}\right),
\end{aligned}
$$

where $v_{\mathrm{F}, \mathrm{e} / \mathrm{h}}$ is the Fermi velocity of electrons/holes at Fermi momentum in the conduction/valence band. Let us note that we have adopted single-particle states to treat random phase approximation using the dielectric function (9) which is calculated based on the single-particle states obtained in the previous section. The results of these calculations for single-particle states are shown in the figures and will be discussed in the forthcoming arguments.

In the one-loop GW approximation with dynamically screened interaction one has $[8,33]$ :

$$
\begin{aligned}
& \Sigma_{i}(k, z)=-\frac{1}{\beta} \sum_{k^{\prime}, z^{\prime}} V_{\mathrm{s}}\left(k-k^{\prime}, z-z^{\prime}\right) G_{i}\left(k^{\prime}, z^{\prime}\right)= \\
& -\frac{1}{\beta} \sum_{k^{\prime}, z^{\prime}} \frac{V_{\mathrm{c}}\left(k-k^{\prime}\right)}{\varepsilon\left(k-k^{\prime}, z-z^{\prime}\right)} G_{i}\left(k^{\prime}, z^{\prime}\right),
\end{aligned}
$$

where

$$
G_{i}(k, z)=\frac{1}{z-\varepsilon_{i, k}-\Sigma_{i}(k, z)+\mu_{i}}, \quad i=\mathrm{e}, \mathrm{h}
$$

and $\Sigma_{\mathrm{e} / \mathrm{h}}(k, z)$ is the self-energy of electrons/holes defined in GW approximation. To avoid multipole structure in the $G_{i}(k, z)$, we approximate $\Sigma_{i}(k, z)$ by the momentum-dependent bandgap renormalization $\Delta_{i}(k)$. Using the approximation $\Delta_{i}(k)=\Sigma_{i}\left(k, \varepsilon_{i, k}-\mu_{i}\right)$, we find the following single pole electron-hole Green function [8, 33]:

$$
G_{i}(k, z) \sim \frac{1}{z-\varepsilon_{i, k}-\Delta_{i}(k)+\mu_{i}}, \quad i=\mathrm{e}, \mathrm{h} .
$$

The above formalism provides a suitable framework for our numerical calculation of bandgap renormalization. To proceed further, we should calculate the screened Coulomb potential. We emphasize that all numerical calculations for dielectric function, screened Coulomb potential and the bandgap renormalization are based on the single-particle states calculated numerically in Sect. 2. Now, using Eq. (5), the screened Coulomb potential can be written as follows:

$$
\begin{aligned}
& V_{\mathrm{c}}(k)=\frac{2 e^{2}}{\varepsilon_{0}} \int \mathrm{d} x \mathrm{~d} y \int \mathrm{d} x^{\prime} \mathrm{d} y^{\prime} K_{0}|k R \| \Psi(x, y)|^{2} \\
& \quad \times\left|\Psi\left(x^{\prime}, y^{\prime}\right)\right|^{2},
\end{aligned}
$$

or using rescaled coordinates $\tilde{x_{i}} \equiv x_{i} / L$ and ${\tilde{x_{i}}}^{\prime} \equiv x_{i}^{\prime} / L$ we find

$$
\begin{aligned}
& V_{\mathrm{c}}(k)=\lambda \frac{2 e^{2}}{\varepsilon_{0}} L^{4} \int \mathrm{d} \tilde{x} \mathrm{~d} \tilde{y} \int \mathrm{d} \tilde{x}^{\prime} \mathrm{d} \tilde{y}^{\prime} K_{0}|k R \| \Psi(\tilde{x}, \tilde{y})|^{2} \\
& \quad \times\left|\Psi\left(\tilde{x}^{\prime}, \tilde{y}^{\prime}\right)\right|^{2}
\end{aligned}
$$

where $\lambda$ is rescaling factor equal to $10^{-18} / \mathrm{m}^{2}$ and $k R=$ $k \sqrt{\left(x-x_{0}\right)^{2}+\left(y-y_{0}\right)^{2}}$. Using $\Psi(x, y)$ computed numerically in the previous section, we solve the integral of Eq. (13). The screened potential is calculated using the ground state wave function as a function of $k$. Figure 11 shows the result of this calculation for different values of the relative width of the confinement potential. In this figure, $V_{\mathrm{c}}(k)$ is normalized by $2 e^{2} / \varepsilon_{0}$ and the $k$ is normalized to $k L$.

In the next step we calculate the bandgap renormalization in both quasi-static and GW approximation for this setup. To do this end, we should calculate dielectric func- 
tion, single-pole Green function for electron and Green function for hole numerically. To calculate these quantities we use single-particle state presented in Sect. 2. In addition, we use the rescaled quantities $\tilde{k}=k L$ and $\omega=\tilde{\omega} \times 10^{16}$. To calculate bandgap renormalization in this configuration, we define the rescaled $\beta_{\mathrm{e}}$ and $\mu_{\mathrm{e}}^{0}$, respectively, as $\beta \hbar^{2} / 2 m_{\mathrm{e}}^{*} L^{2}=\beta\left(574.5 / L^{2}\right)$ and $\mu_{\mathrm{e}}^{0} \beta$. For simplicity and without loss of generality, we can set $L=W_{y}$ where $W_{y}$ is the width of the quantum well in the $y$ direction in $\mathrm{nm}$. For holes, we also define the rescaled $\beta_{\mathrm{h}}$ and $\mu_{\mathrm{h}}^{0}$ as $\beta_{\mathrm{h}}=\beta_{\mathrm{e}}\left(\frac{m_{\mathrm{e}}^{*}}{m_{\mathrm{h}}^{*}}\right)$ and $\mu_{\mathrm{h}}^{0}=\mu_{\mathrm{e}}^{0}\left(\frac{m_{\mathrm{e}}^{*}}{m_{\mathrm{h}}^{*}}\right)$, respectively. In all of our computations in this paper, we have assumed that the ratio $m_{\mathrm{h}}^{*} / m_{\mathrm{e}}^{*}$ is equal to 0.3 and $m_{\mathrm{e}}^{*} \approx 0.067 m_{\mathrm{e}}$. Then we set $\tilde{\omega}=0$ and using Eq. (7), we calculate the bandgap renormalization in both quasi-static and GW approximations at temperature $T=0$. The results of these calculations for both quasi-static and GW approximations are shown in Fig. 12. As this figure shows, the absolute values of the bandgap renormalization in GW approximation are smaller than in the case with quasi-static plasmon-pole approximation. Typical values of bandgap normalization are in the range of 10$30 \mathrm{meV}$ depending on the temperature and impurities in the system. Because of consideration of more quantum field theoretical details, GW approximation generally gives results which have better agreement with experimental results $[10,11]$. In fact, generally the dynamical correlation effects tend to reduce the magnitude of the bandgap renormalization, especially when compared with the quasi-static approximation results, and bring the calculated values to be closer to the experimental results.

\section{Results and discussions}

Probability distribution of charge carriers in the absence of the external electric and magnetic fields are shown in Fig. 2 where the asymmetric parameter $a=$ $W_{x} / W_{y}$ has been set equal to 0.8 . This figure emphasizes the central role played by the geometric shape of the confinement potential. In the absence of the external electric and magnetic fields, carrier distribution obeys the symmetry of the confinement potential. Variation of asymmetric parameter changes the profile of the charge distribution in such a way that the case with $a=1$ has maximum symmetry and any change of the relative width leads to the antisymmetric distribution. Figure 3 shows the variation of the ground state energy of charge carriers versus the inverse of the asymmetric parameter $a$. Variation of the relative width leads to the conclusion that smaller relative width leads to smaller ground state energy. Now, let us suppose that we turn on a uniform magnetic field. In the absence of electric field, the distribution of charge carriers is given by Fig. 4 . The role played by the asymmetric parameter is a reduction of the probability amplitude when the width of the well increases in $x$ or $y$ direction. Variation of the ground state energy versus the intensity of the magnetic field is depicted in
Fig. 5 for two different values of the asymmetric parameter. For a fixed well width in the $y$ direction, when the width of the well in the $x$ direction increases, the value of the ground state energy will increase. Now we turn off magnetic field and apply a uniform electric field in the $x$ direction. The profile of probability amplitude for charge carriers distribution is shown in Fig. 6 for $a=0.8$. Figure 7 shows the cross-section of this distribution from top view. The probability amplitude has a Gaussian profile and is shifted toward the right hand side of the well. This shift is a function of the electric field intensity. The probability profile does not obey the geometric shape of the confinement potential due to preferred direction defined by the presence of the electric field. The energy of the ground state versus the intensity of the electric field is depicted in Fig. 8. As this figure shows, ground state energy decreases with increasing the electric field intensity. This is not surprising since external electric field tends to decouple electrons and holes from each other leading to less bounded exciton state. In the general case where both electric and magnetic fields are present, the asymmetric behavior explained above will be enhanced in some respects. Figure 9 shows the space variation of the probability amplitude with $a=0.8$. In the presence of the constant electric and magnetic fields, the pick of the graph describing charge carriers distribution shifts to the positive direction of the $x$ axis. This feature causes the carriers concentration in such a way that these carriers distributions do not obey the external confinement potential symmetry. What is more, the intensity of the electric field results in the larger shift of the distribution peak to the right hand side of the $x$ axis. The presence of the constant magnetic field causes the anisotropy in the profile of the probability distribution of the carriers. Figure 10 shows the variation of the ground state energy versus the variation of the electric field when the magnetic field is supposed to be constant. More intensity of the electric field leads to the more reduction of the bound states energies. This resembles the linear Stark effect in the elementary quantum mechanics. In the language of many-body effects in the dense plasma within a quasi-one-dimensional semiconductor, application of the intense electric field results in the weaker excitonic states. Therefore, the presence of the external electric and magnetic fields will shift the location of the maximum concentration of carriers and in this case there is an apparent asymmetry in the profile of carriers distribution. This point can be used in fabrication of microelectronic devices based on quantum wires.

As another step, we have proposed a numerical framework to calculate the screened Coulomb potential and the values of the bandgap energy renormalization in this H-shaped quantum wire within two different approximations: quasi-static and dynamical random phase approximation in its leading order dynamical screening (GW) approximation. We have evaluated the single-particle self-energies for both electrons and holes in the dynamical plasmon-pole approximation (PPA) and the 
726

K. Nozari et al.

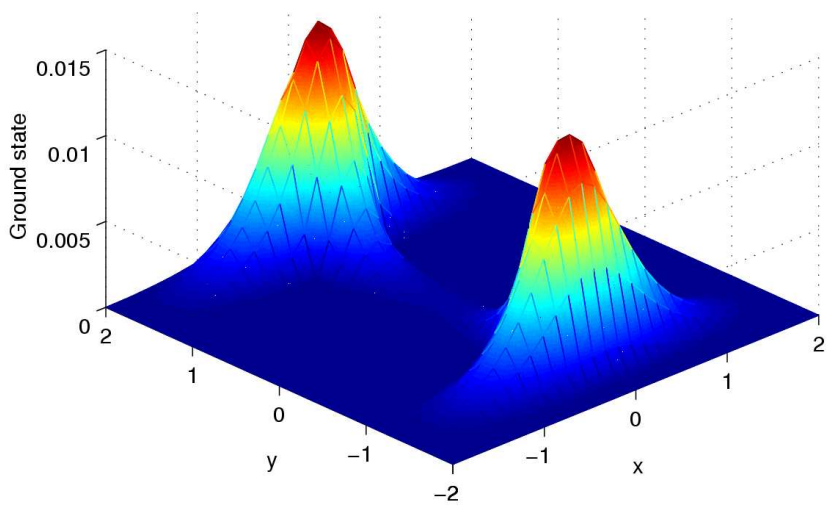

Fig. 2. The probability amplitude of charge carriers distribution for ground state of $\mathrm{H}$-shaped quantum wire at zero external EM fields. The asymmetry parameter was chosen to be $a=W_{x} / W_{y}=0.8$.

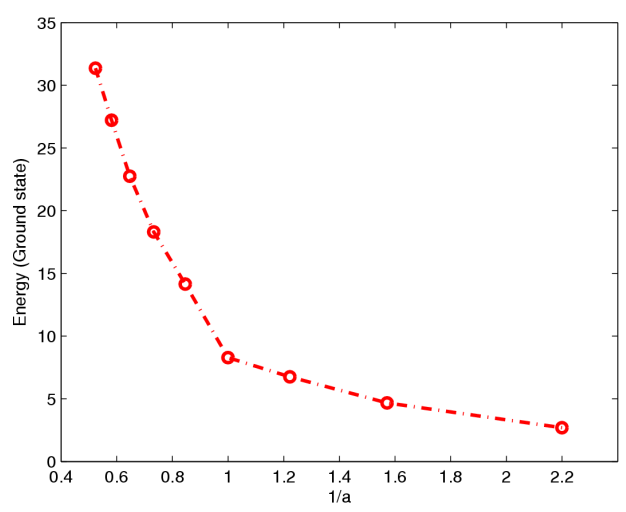

Fig. 3. The absolute values of the ground state energy (in $\mathrm{eV}$ ) versus the inverse of the asymmetry parameter at zero external fields strength.

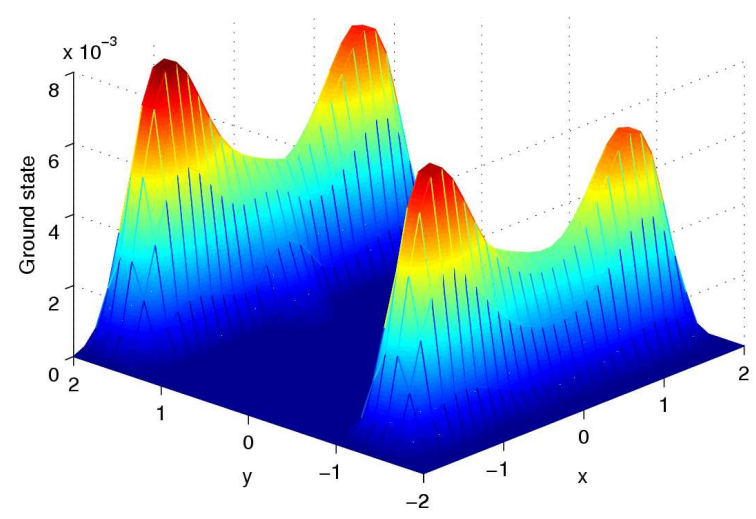

Fig. 4. The probability amplitude of charge carriers distribution for ground state of $\mathrm{H}$-shaped quantum wire in the presence of an external magnetic field (and zero electric field). The asymmetry parameter was chosen to be $a=W_{x} / W_{y}=0.8$.

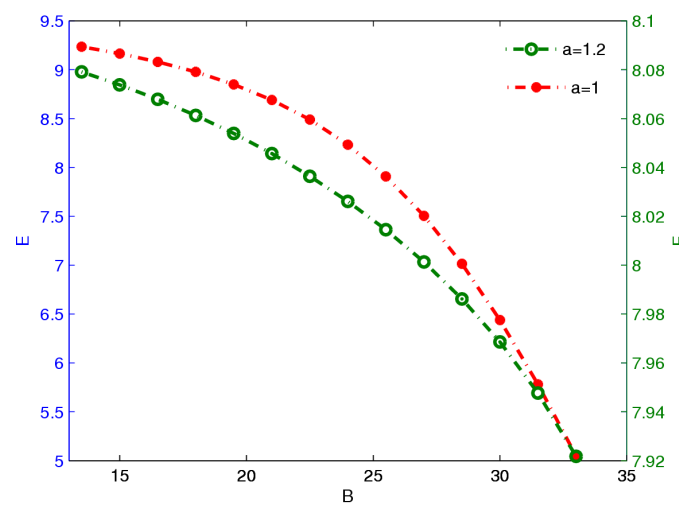

Fig. 5. The absolute values of the ground state energy versus the intensity of the external magnetic field for different values of asymmetry parameter.

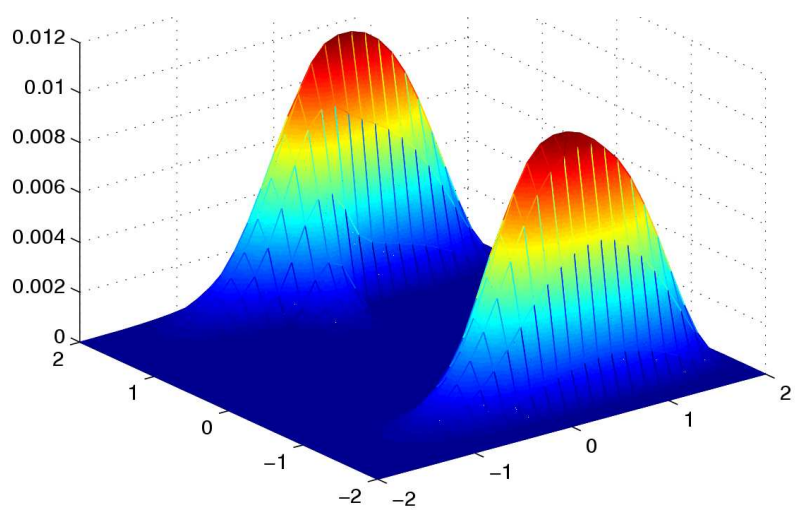

Fig. 6. The probability amplitude of charge carriers distribution for ground state of $\mathrm{H}$-shaped quantum wire in the presence of an external electric field (and zero magnetic field). The asymmetry parameter was chosen to be $a=0.8$.

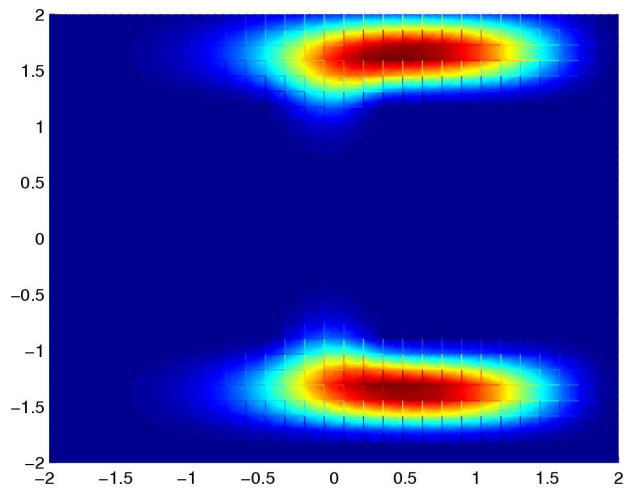

Fig. 7. The cross-section of the probability amplitude of charge carriers distribution for ground state of $\mathrm{H}$-shaped quantum wire in the presence of an external electric field (and zero magnetic field). The asymmetry parameter was chosen to be $a=0.8$ and distances are in nanometers. 


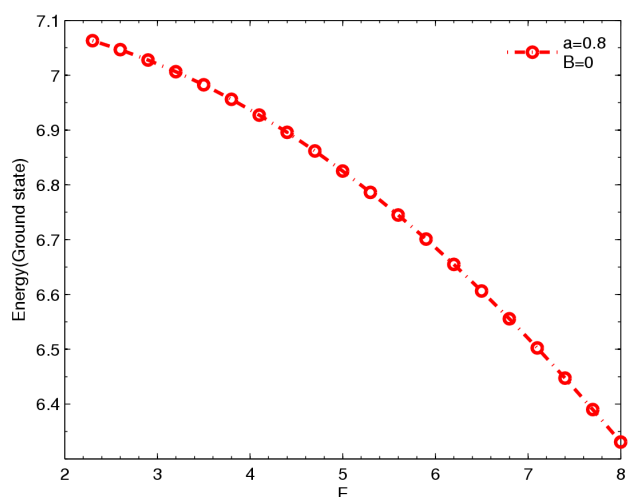

Fig. 8. The absolute values of the ground state energy versus the intensity of the external electric field for $a=0.8$ and $B=0$.

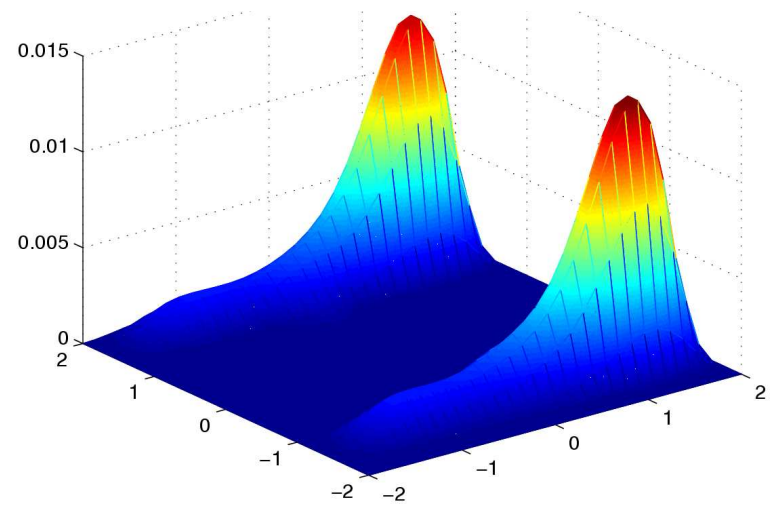

Fig. 9. The probability amplitude of charge carriers distribution for ground state of $\mathrm{H}$-shaped quantum wire in the presence of both electric and magnetic fields. The asymmetry parameter was chosen to be $a=0.8$.

leading order dynamically screened interaction or GW approximation to obtain the electron and hole renormalized Green functions. This self-energy calculation gives us the band gap renormalization due to exchange-correlation effect. For comparison, we also calculated the bandgap renormalization obtained by the quasi-static calculation in both static random phase approximation and static plasmon-pole approximation. Let us note that quasi-static approximation works well in two- and three-dimensional systems but fails completely in one-dimensional systems. This is the case because the electrons in one-dimensional system suffer very strong inelastic scattering effects by virtue of the restricted phase space. However, we have used this approximation only for comparison purposes. It is important to note that by rescaling procedure we have adopted here, we have fixed the geometry of the H-shaped quantum wire. Actually, one should consider the possibility for variation of the geometrical shape, too. However, we have noticed that by a change in the geometrical shape via variation of the parameter $L=W_{y}$ has no considerable effect on the

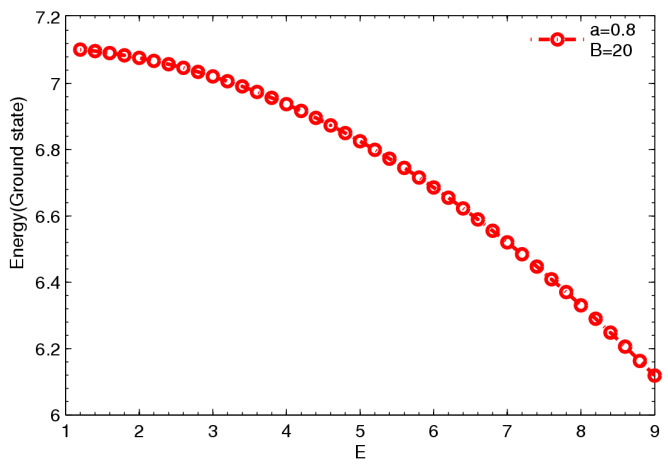

Fig. 10. The absolute values of the ground state energy versus the intensity of the external electric field for $a=0.8$ and $B=20$ (a constant magnetic field).

main physical results obtained and focused on here. Figure 11 shows the screened Coulomb potential calculated based on the random phase approximation in its leading order and for different values of the asymmetry parameter. Exchange-correlation many-body effects mediate the bare Coulomb interaction. Based on the different width of the confinement potential, screened Coulomb potential varies with geometrical characteristics (such as the relative width) of the confinement potential. As Fig. 11 shows, by increasing the asymmetry parameter, the screened Coulomb potential will grow but its overall behavior with respect to the wave number does not change. Figure 12 shows the calculated band gap renormalization in the quasi-static and dynamical random phase approximations. To calculate bandgap renormalization, we first calculated the electron/hole single-pole Green function and then using the formalism of both one-loop GW approximation and quasi-static plasmon-pole approximation we calculated the values of the bandgap renormalization in temperature $T=0$. Let us note that we have considered $L$ dependence of the bandgap renormalization, however it can be translated to bandgap renormalization versus carrier densities as well. Figure 12 also compares the results of the bandgap renormalization in GW and plasmon-pole approximations. As this figure shows, for a fixed value of $L, \mathrm{GW}$ approximation gives smaller absolute values of the band gap renormalization. Generally, GW approximation gives more reliable result in comparison with experimental data [5].

Let us note also that we have focused on the variation of the geometrical shape of the confinement potential by variation of $L$. As the figures (for instance Fig. 1) show, there are other possibilities for variation of the geometrical shape of the external confinement potential. However, the general behaviors are the same as presented here. There are some restrictions on our calculations which can be summarized as follows: the many-body treatment has the disadvantage that, for bandgap renormalization, it commonly ignores geometrical factors such as the quantum confined Stark effect whose relevance is structure specific. In other words, the numerical results for differ- 


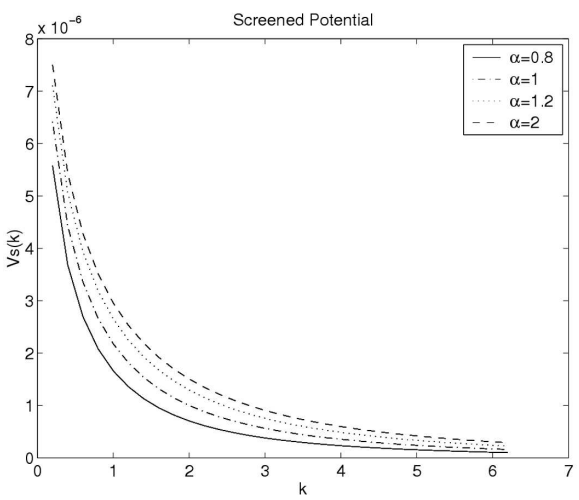

Fig. 11. The calculated screened Coulomb potential versus the wave number for different asymmetry parameter. The screened potential is normalized by $2 e^{2} / \varepsilon_{0}$ and the $k$ is normalized by $L=W_{y}$.

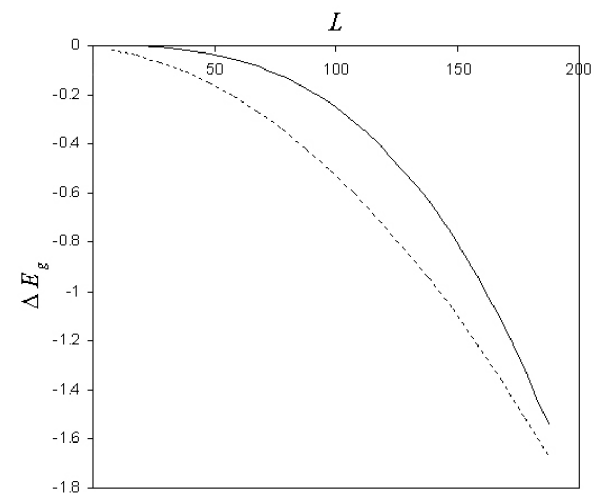

Fig. 12. Calculated band gap renormalization in H-shaped quantum wire within dynamical random phase (GW) approximation (upper curve) and quasi-static plasmon-pole approximation (lower curve). The screened potential is normalized by $2 e^{2} / \varepsilon_{0}$ and $L=W_{y}$.

ent types of the confinement potential of the H-shaped quantum wire may be geometry dependent in general. Furthermore, in the exciton problem, many-body theory treats screening within the linear approximation and, generally, influence of the bound electron on the free electrons is not fully included. In particular, the orthogonality of the free electron states with the bound state, which increases its importance in lower dimensional systems, are typically not included. On the other hand, the complete treatment of the problem should consider the effects of several subbands [8]. Let us note that in the rest of the calculation of bandgap renormalization presented here, we have used the two-band (one conduction band and one valence band) model and we have neglected the effects of higher subbands and degeneracies in valence bands. We also assumed that electrons and holes densities are constant in time. We are going to include the effect of higher excitations in the bandgap renormalization in our forthcoming study. Also, we shall study the effect of external electric and magnetic fields on the values of the bandgap renormalization in our future study. These are important issues which need more attention and there is an explicit gap in literature in this field.

\section{Summary and conclusions}

Our numerical procedure to study bound states and many-body effects in the $\mathrm{H}$-shaped quantum wire structure has the following results:

- The distribution of the probability of the charge carriers in the ground state of the H-shaped quantum wire in the absence of electric and magnetic fields has a symmetric shape obeying the geometric shape of the confinement potential. This distribution has its maximum at the center of each arm and decreases with distance from the center. The relative width of the confinement potential, the asymmetric ratio $a=W_{x} / W_{y}$ has considerable effect on the profile of this distribution.

- In the presence of a constant magnetic field, the distribution of the charge carriers becomes oscillatory both in $x$ and $y$ directions. Increasing the strength of the magnetic field leads to the reduction of the ground state energy. The role played by the asymmetric parameter is given by the reduction of the probability amplitude when the width of the well increases in the $x$ or $y$ directions.

- The situation for the case of non-vanishing electric field (in the absence of the magnetic field) resembles the Stark effect in a low dimensional system. The probability amplitude has an oscillatory behavior with larger wavelength of the oscillations. In this case although the probability amplitude has a Gaussian profile, it is shifted toward the right hand side of the well shown in Fig. 1. This shift is a function of the electric field intensity. The probability profile does not obey the symmetry of the geometric shape of the confinement potential due to the preferred direction defined by the presence of the electric field.

- In the presence of both electric and magnetic fields there are oscillations in probability distribution both in $x$ and $y$ directions, but in this case the probability distribution is not symmetric. In the presence of constant electric and magnetic fields, the peak value of the probability amplitude will be shifted toward the positive direction of the $x$ axis. This causes the carriers to be concentrated in such a way that they do not obey the external confinement potential symmetry. Larger intensity of the electric field results in larger shift of the distribution peak to the right hand side of the $x$ axis. The presence of the constant magnetic field causes an anisotropy in the profile of the probability distribution of the carriers, since it apparently breaks 
the local rotational symmetry in the center of each arm.

- Screened Coulomb potential of the H-shaped external confinement is a sensitive function of the asymmetry parameter but its overall behavior under variation of the wave number is the same for other possible geometries of quasi-one-dimensional systems. The calculated bandgap narrowing for the $\mathrm{H}$-shaped confinement potential in the absence of the electric and magnetic fields and within quasi-static and dynamical random phase approximation shows a typical gap narrowing of the order of few $\mathrm{meV}$. This is supported from other studies of band gap narrowing in quasi-one-dimensional semiconductor systems $[4,6]$. We have shown that GW approximation gives smaller absolute values of the bandgap renormalization. The dynamical random phase GW approximation leads to more reliable result than quasi-static approximation in comparison with experiments.

- Our analysis has some restrictions that should be considered in a more complete study: essentially, the many-body treatment has the disadvantage that for bandgap renormalization it commonly ignores geometrical factors such as the quantum confined Stark effect which is a structure dependent effect. In our study, the influence of the bound electrons on the free electrons is not fully included in the analysis. A complete treatment of the problem should consider the effects of several subbands. We have neglected the effects of higher subbands and degeneracies in valence bands. We also assumed that electrons and holes densities are constant in time. In our forthcoming paper which will be reported soon, we will include the effect of higher excitations in the bandgap renormalization and we shall study the effect of external electric and magnetic fields on the values of the bandgap renormalization. These are important issues which need more attention and there is an explicit gap in literature in this respect.

In summary, we can conclude that in the presence of electric and magnetic fields, H-shaped quantum wires bound states characteristics are sensitive functions of an asymmetric parameter $a=W_{x} / W_{y}$ and the strength of the electric and magnetic fields. The case of non-vanishing electric and magnetic fields induces an intrinsic inhomogeneity in the quasi-one-dimensional system. Many-body effects due to plasma screening and resulting optical nonlinearities are also dependent on the asymmetric parameter of quasi-one-dimensional confinement potential. Among these nonlinear optical effects, bandgap renormalization of fundamental band edge has been studied numerically in this paper. The absolute values of the bandgap renormalization in GW approximation are smaller than the quasi-static plasmon-pole result. Typical values of this normalization are few meV depending on the temperature and impurities in the system. GW approximation generally gives results which have better agreement with experiment.

\section{Appendix: numerical strategy}

We use the finite difference algorithm (see for instance [34]) to solve our partial differential Eq. (2) with boundary conditions imposed by H-shaped confinement potential as defined in relation (3). The most straightforward refinement method replaces the differential equation with a finite difference equation. It replaces all derivatives with approximate expression as the following familiar form:

$$
\begin{aligned}
& \left(\frac{\mathrm{d} y}{\mathrm{~d} x}\right)_{x=x_{n}} \approx \frac{y_{n+1}-y_{n}}{h}, \\
& \left(\frac{\mathrm{d}^{2} y}{\mathrm{~d} x^{2}}\right)_{x=x_{n}} \approx \frac{y_{n+1}-2 y_{n}+y_{n-1}}{h^{2}},
\end{aligned}
$$

where the mesh chosen to be equally spaced and given by $h=\frac{x_{k}-x_{0}}{N+1}$ with $x_{k}=x_{0}+k h$ and $k=1,2, \ldots, n+1$. We first rescale the Schrödinger equation and the confinement potential. We define the rescaled value of $x$ and $y$ as $\bar{x} \equiv x / W_{x}$ and $\bar{y} \equiv y / W_{y}$ where $W_{x}$ and $W_{y}$ are the width of the well in the $x$ and $y$ directions, respectively. Now the rescaled Schrödinger equation can be written as follows:

$$
\begin{aligned}
& -\left(\frac{1}{W_{x}} \frac{\partial^{2}}{\partial \bar{x}^{2}}+\frac{1}{W_{y}} \frac{\partial^{2}}{\partial \bar{y}^{2}}-\frac{2 \mathrm{i} e B W_{x}}{\hbar W_{y}} \bar{x} \frac{\partial}{\partial \bar{y}}\right) \Psi(\bar{x}, \bar{y}) \\
& +\left(\frac{e B W_{x}}{\hbar}\right)^{2} \bar{x}^{2} \Psi(\bar{x}, \bar{y}) \\
& -\frac{2 m e E W_{x}}{\hbar^{2}} \bar{x} \Psi(\bar{x}, \bar{y})=\frac{2 m}{\hbar^{2}} \lambda \Psi(\bar{x}, \bar{y}) .
\end{aligned}
$$

Using the finite difference algorithm, this equation can be written as follows:

$$
\begin{aligned}
& -\left(\frac{\Psi_{i+1, j}-2 \Psi_{i, j}+\Psi_{i-1, j}}{\Delta_{x}}\right. \\
& \left.+a^{2} \frac{\Psi_{i, j+1}-2 \Psi_{i, j}+\Psi_{i, j-1}}{\Delta_{y}}\right) \\
& +\frac{2 \mathrm{i} e a B W_{x}^{2}}{\hbar} \bar{x}_{i} \frac{\Psi_{i, j+1}-\Psi_{i, j-1}}{2 \Delta_{y}} \\
& +\left(\frac{e B W_{x}^{2}}{\hbar}\right)^{2} \bar{x}_{i}^{2} \Psi_{i, j}-\frac{2 m e E W_{x}^{3}}{\hbar^{2}} \bar{x}_{i} \Psi_{i, j} \\
& =\frac{2 m W_{x}^{2}}{\hbar^{2}} \lambda \Psi_{i, j},
\end{aligned}
$$

where $a=W_{x} / W_{y}$ is the relative width of the H-shaped quantum wire. We do discretize the $x$ and $y$ axes to discrete space $\mathrm{d} x=0.1$ and $\mathrm{d} y=0.1$, therefore Eq. (16) can be written as a matrix equation 
$\mathcal{H} \phi=E \phi$,

where $\mathcal{H}$ and $\phi$ are the Hamiltonian matrix and the state wave functions array which are defined as follows:

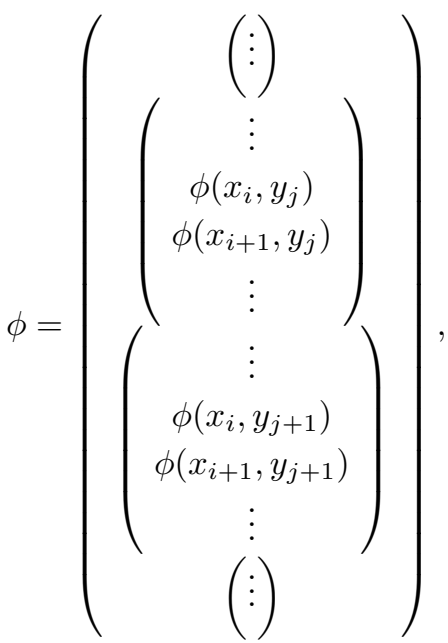

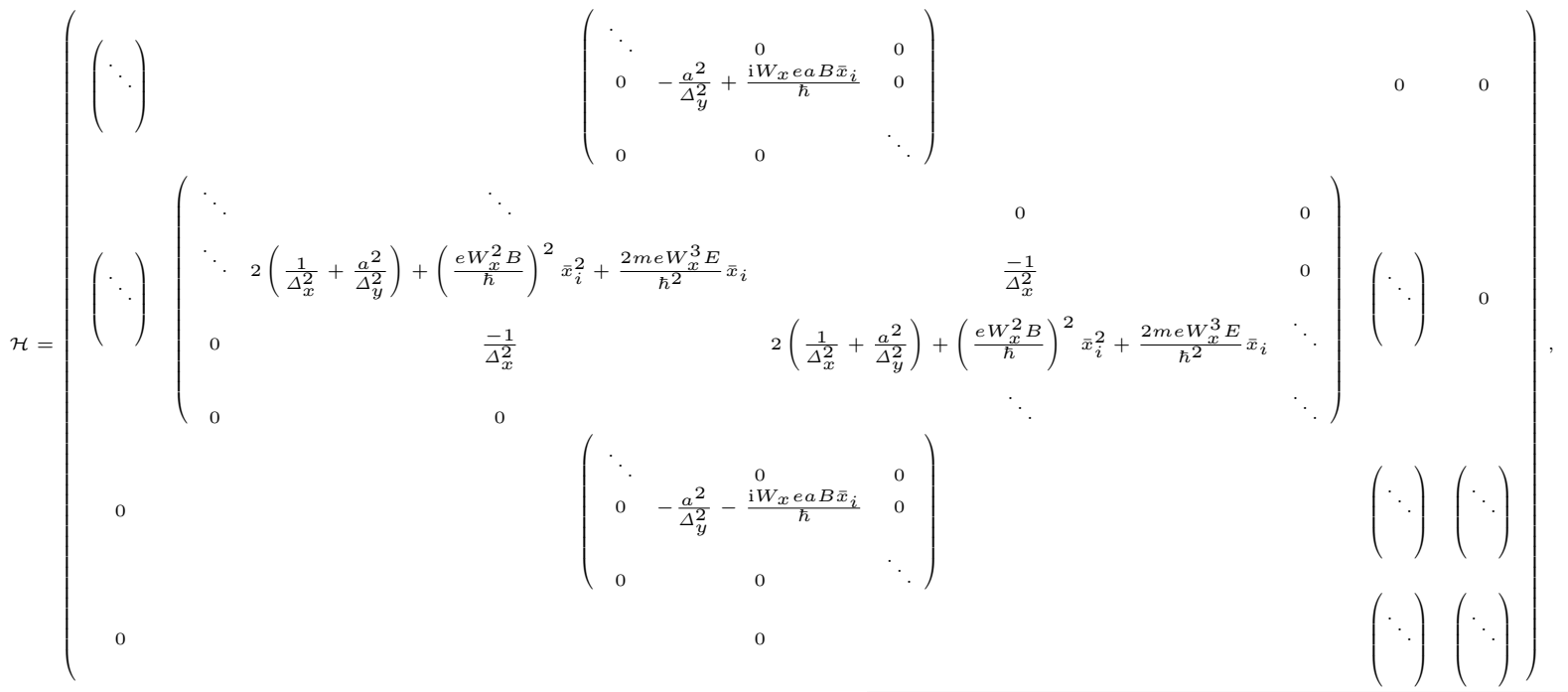

The error of computation of $\phi(x, y)$ is of the order of $\mathrm{O}\left(\mathrm{d} x^{2}\right)$. We diagonalize the Hamiltonian matrix and calculate the bound states energies and wave functions numerically. We use MATLAB package since it uses techniques that are more efficient than the Jacobi rotations and that can be applied to asymmetric or even complex matrices as well as to the more common real symmetric situations. The result of these numerical calculations of single-particle state are used in the calculation of the dielectric function, screened confinement potential and the bandgap renormalization.

\section{References}

[1] N. Peyghambarian, S.W. Koch, A. Mysyrowicz, Introduction to Semiconductor Optics, Prentice Hall, New Jersey 1993.
[2] H. Haug, S.W. Koch, Quantum Theory of the Optical and Electronic Properties of Semiconductors, World Sci., Singapore 1993.

[3] V.V. Mitin, V.A. Kochelap, M.A. Stroscio, Quantum Heterostructures: Microelectronics and Optoelectronics, Cambridge University Press, Cambridge (UK) 1999.

[4] P. Harrison, Quantum Wells, Wires and Dots, Wiley, 2000 .

[5] H. Kalt, Optical Properties of III-V Semiconductors, Springer-Verlag, Berlin 1996.

[6] B.Y. Hu, S. Das Sarma, Phys. Rev. B 48, 5469 (1993).

[7] G. Goldoni, F. Rossi, E. Molinari, Phys. Rev. B 55, 7110 (1996).

[8] D.W. Wang, S. Das Sarma, Phys. Rev. B 64, 195313 (2001).

[9] S. Das Sarma, D.W. Wang, Phys. Rev. Lett. 84, 2010 (2000). 
[10] E.H. Hwang, S. Das Sarma, Phys. Rev. B 58, R1738 (1998).

[11] S. Das Sarma, E.H. Hwang, Phys. Rev. B 59, 10730 (1999).

[12] R. Rinaldi, R. Cingolani, in: Optical Spectroscopy of Low Dimensional Semiconductors, Eds. G. Abstreiter, A. Aydinli, J.-P. Leburton, Kluwer Academic, Dordrecht 1997.

[13] S. Benner, H. Haug, Europhys. Lett. 16, 579 (1991); Phys. Rev. B 47, 15570 (1993).

[14] B. Tanatar, in: Optical Spectroscopy of Low Dimensional Semiconductors, Eds. G. Abstreiter, A. Aydinli, J.-P. Leburton, Kluwer Academic, Dordrecht 1997.

[15] B. Tanatar, J. Phys., Condens. Matter 8, 5997 (1996).

[16] K. Güven, B. Tanatar, C. R. Bennett.J. Phys. Condens. Matter 12, 2031 (2000).

[17] S. Bellucci, P. Onorato, Eur. Phys. J. B 47, 385 (2005).

[18] S. Bellucci, P. Onorato, Eur. Phys. J. B 45, 87 (2005).

[19] S. Bellucci, P. Onorato, Phys. Rev. B 73, 045329 (2006).

[20] S. Bellucci, P. Onorato, Phys. Rev. B 74, 245314 (2006).

[21] S. Bellucci, P. Onorato, Eur. Phys. J. B 52, 469 (2006).
[22] S.S.Z. Ashraf, A.C. Sharma, J. Phys. Condens. Matter 17, 3043 (2005).

[23] S. Sedlmaier, M. Stopa, G. Schedelbeck, W. Wegscheider, G. Abstreiter, Phys. Rev. B 65, 201304 (2002); S. Sedlmaier, M. Stopa, G. Schedelbeck, W. Wegscheider, G. Abstreiter, arXiv:cond-mat/9910002.

[24] M. Stopa, Phys. Rev. B 63, 195312 (2001).

[25] M.H. Szymanska, P.B. Littlewood, R.J. Needs, Phys. Rev. B 63, 205317 (2001).

[26] Y.-K. Lin, Y.-N. Chen, D.-S. Chuu, Phys. Rev. B 64, 193316 (2001).

[27] K. Nozari, M. Madadi, Phys. Scr. 69, 244 (2004).

[28] K. Nozari, M. Madadi, Phys. Scr. 73, 1 (2006).

[29] Y. Lin, K. Lin, D. Chuu, arXiv:cond-mat/0306361.

[30] M. Shin, S. Lee, K.W. Park, E.-H. Lee, Superlattices Microstruct. 16, 147 (1994).

[31] E.M. Hankiewiczm, L.W. Molenkamp, T. Jungwirth, Jairo Sinova, Phys. Rev. B 70, 241301(R) (2004).

[32] O.P. Arhaug, O. Soeraasen, J. Phys. Conf. Series 34, $686(2006)$

[33] G.D. Mahan, Many-Particle Physics, 3rd ed., Kluwer Academic/Plenum, New York 2000.

[34] W.H. Press, B.P. Flannery, S.A. Teukolsky, W.T. Vetterling, Numerical Recipes - The Art of Scientific Computing, Cambridge, New York 1989 